\title{
BMJ Open Epidemiology of depressive disorders in people living with hypertension in Africa: a systematic review and meta- analysis
}

\author{
Francky Teddy Endomba, ${ }^{1,2}$ Temgoua Ngou Mazou (D) , ${ }^{1}$ Jean Joel Bigna (D) ${ }^{3}$
}

To cite: Endomba FT, Mazou TN Bigna JJ. Epidemiology of depressive disorders in people living with hypertension in Africa: a systematic review and meta-analysis. BMJ Open 2020;10:e037975. doi:10.1136/ bmjopen-2020-037975

- Prepublication history and supplemental material for this paper is available online. To view these files, please visit the journal online (http://dx.doi org/10.1136/bmjopen-2020037975).

Received 23 February 2020 Revised 12 November 2020 Accepted 17 November 2020

A) Check for updates

(C) Author(s) (or their employer(s)) 2020. Re-use permitted under CC BY-NC. No commercial re-use. See rights and permissions. Published by BMJ.

${ }^{1}$ Health Economics and Policy Research and Evaluation for Development Results Group, Yaounde, Cameroon

${ }^{2}$ Psychiatry Internship Program, Université de Bourgogne, Dijon, Bourgogne, France

${ }^{3}$ Department of Epidemiology and Public Health, Centre

Pasteur du Cameroun, Yaounde, Cameroon

Correspondence to

Dr Jean Joel Bigna;

bignarimjj@yahoo.fr

\section{ABSTRACT}

Objectives Better knowledge of epidemiology of depressive disorders in people living with hypertension can help to implement pertinent strategies to address its burden. The objective was to estimate the prevalence of depressive disorders and symptoms in people living with hypertension in Africa.

Design Systematic review and meta-analysis.

Data sources PubMed, EMBASE, African Index Medicus, African Journals OnLine were searched up to 31 January 2020, regardless of the language of publication.

Eligibility criteria We included studies conducted among adult patients with hypertension ( $\geq 18$ years) living in Africa and reporting the prevalence of depressive disorders and symptoms.

Data extraction and synthesis Two independent investigators selected studies, extracted data and assessed the methodological quality of included studies by using the tools developed by Joanna Briggs Institute. Multivariate random-effects meta-analysis served to pool data by considering the variability between diagnostic tools used to identify patients with depressive disorders or symptoms.

Results We included 11 studies with 5299 adults with hypertension. Data were collected between 2002 and 2017, from South Africa, Nigeria, Ghana, Ethiopia and Burkina Faso. The mean age varied between 50.3 years and 59.6 years. The proportion of men ranged from $28 \%$ to $54 \%$. The adjusted prevalence of depressive disorders taking into account the variance between diagnostic tools was $17.9 \%$ (95\% Cl $13.0 \%$ to $23.4 \%$ ). The prevalence of depressive symptoms and major depressive symptoms was $33.3 \%$ (95\% Cl $9.9 \%$ to $61.6 \%$ ) and $7.8 \%$ (95\% Cl $3.0 \%$ to $14.5 \%$ ), respectively. There was heterogeneity attributable to the diagnostic tools for depressive disorders and symptoms. There was no publication bias.

Conclusion Notwithstanding the representativeness lack of some (sub) regions of Africa, weakening the generalisability of findings to the entire region; depressive disorders and symptoms are prevalent in people living with hypertension in Africa, indicating that strategies from clinicians, researchers and public health makers are needed to reduce its burden in the region.
Strengths and limitations of this study

- Not all subregions of Africa were represented in this review.

- This is the first review performed among people living with hypertension in Africa to investigate the epidemiology of depressive disorders and symptoms.

- We found a huge heterogeneity between studies explained by the difference between diagnostic tools for depressive disorders.

- We were not able to explore all sources of heterogeneity due to scarcity of epidemiological data.

\section{INTRODUCTION}

Cardiovascular diseases (CVDs) are the leading cause of deaths worldwide with approximately 18 million deaths per year. ${ }^{1}$ Hypertension is involved in approximately $50 \%$ of CVDs. ${ }^{2-4}$ According to the WHO, the number of people living with hypertension worldwide is estimated at 1.13 billion, and Africa has the highest prevalence of people with hypertension (27\%). ${ }^{4}$ In addition to high morbidity and mortality, hypertension is also associated with high socioeconomic burden related to its management and complications. ${ }^{5-7}$ Detrimental outcomes related to chronic high blood pressure encompass target organ damage involving cerebrovascular, heart and kidney diseases, ${ }^{45}$ and mental health repercussions including anxiety, stress and depression. ${ }^{8-10}$

Depressive disorder is the most common mental health disease and the second cause of disability worldwide after CVDs. ${ }^{1112}$ It accounts for $3 \%$ of the global disability adjusted life years. ${ }^{12}$ According to the WHO, depressive disorder affects more than 300 million people $(4.4 \%$ of the global population), and its prevalence in the African continent is estimated at 9\% (29.9 million cases) in the general population. ${ }^{13}$ Some subpopulations have been identified as at higher risk of 
depression, including patients with chronic CVDs such as hypertension. ${ }^{914} \mathrm{~A}$ wide range of previously published studies addressed the interaction between hypertension and depression. ${ }^{8101516}$ Most of these studies concluded that hypertension and depressive disorder share bidirectional interplay where patients with hypertension were more likely to develop depression and conversely, those with depression have an increased risk to develop hypertension. ${ }^{817}$ As examples for evidence, a meta-analysis of 41 studies (including 31 studies from China and 3 from Africa) which included 30796 patients with hypertension found that $26.8 \%$ have depression, and another one which included 22367 participants found that depression significantly increases the risk of hypertension incidence. ${ }^{10} 15$ Additionally, it has been reported that patients living with hypertension with comorbid depression are at higher risk of suboptimal medication adherence with uncontrolled blood pressure, complicated by chronic vascular complications and CVD-related mortality. ${ }^{8} 10151618$ The burden of depression-hypertension co-occurrence is worsened by the fact that approximately 1 patient with hypertension in 10 has untreated depression. ${ }^{10} 19$

Considering up-to-date scientific literature, depression in patients with hypertension is common in western contexts. ${ }^{102}$ Hypertension and depression are commonly encountered among African populations, ${ }^{2}{ }^{13}$ but data summarising and focusing on the burden of depression among hypertensive Africans are not yet available. Hence, we conducted this systematic review and metaanalysis with the aim to explore the prevalence of depressive disorders/symptoms and major depression in people living with hypertension in Africa.

\section{METHODS \\ Design}

This systematic review and meta-analysis was conducted according to the Joanna Briggs Institute guidelines. ${ }^{21}$ This study was reported according to the Preferred Reporting Items for Systematic Reviews and MetaAnalyses guidelines. ${ }^{22}$ The protocol of this review was registered in the International Register of Systematic Reviews (PROSPERO) with the following registration number: CRD42020168979.

\section{Eligibility criteria}

\section{Condition}

We considered studies reporting the prevalence (or enough data to compute this estimate) of depressive disorders and symptoms. We considered depressive disorders (and major depressive disorders) diagnosed according to the Diagnostic and Statistical Manual of Mental Health Disorders IV or V, ${ }^{23}{ }^{24}$ or International Statistical Classification of Diseases and Related Health Problems- $10 .^{25}$ In the studies where depressive disorders were not defined using the aforementioned criteria, we considered the definition used by authors, especially including diagnostic scores such as the Patient Health Questionnaire-9.
Major depressive symptoms notably included depressive mood and anhedonia.

Context

We considered studies conducted in people living in Africa. Studies conducted in Africans living outside Africa were not considered.

\section{Population}

We considered studies conducted in adults ( $\geq 18$ years) living with hypertension regardless of the diagnosis criteria, the therapeutic regimen and the control status for the hypertensive disease.

\section{Study design}

Cross-sectional, case-control and cohort studies.

\section{Data sources}

We searched PubMed, Excerpta Medica Database (EMBASE), African Index Medicus and African Journals OnLine to identify all relevant records published up to 31 January 2020, without any language restriction. The search strategy in EMBASE is available in online supplemental table 1. To supplement the bibliographical database searches and identify potential additional data sources, we scrutinised the reference lists of all relevant original and review papers.

\section{Study selection}

Titles and abstracts of articles retrieved from literature search were independently screened by two investigators (FTE and JJB), and the full texts of those potentially eligible articles were obtained and further assessed for final inclusion. Disagreements were resolved through consensus.

\section{Data collection and management}

A preconceived and standardised data extraction form was used to collect information on the first author's name, study country, year of publication, period of participants' recruitment, study design, setting, sampling method, timing of data collection, response rate, mean or median age of the population, age range, proportion of men, number of participants with hypertension, and number of participants with depressive disorders. In case of multinational studies, data were separated to show the estimate within individual countries. Two investigators (FTE and JJB) independently extracted the data from individual studies, with disagreements being resolved through consensus.

Two investigators (FTE and JJB) independently assessed the methodological quality of the included studies with tools developed by the Joanna Briggs Institute, ${ }^{21}$ with disagreements being resolved through discussion and consensus. Risk of bias was considered low for each criterion if studies used probabilistic sampling, prospectively collected data, had an adequate sample size (required sample size attained), had a response rate $>80 \%$ and used the same method for collection of data of participants. 
Studies with low risk of bias had to have four or more criteria, two or three for moderate risk of bias, and no or one for low risk of bias.

\section{Data synthesis and analysis}

Meta-analyses were performed with the 'meta', 'metafor' and 'dmetar' packages of the statistical software $R$ (V.3.6.2). Prevalence estimates were reported with 95\% CI. Prevalence pooling was done with Freeman-Tukey double arcsine transformation using the random-effects metaanalysis model. ${ }^{26}$ We adjusted the prevalence in a multivariate meta-analysis to take into account the variance between tools used to identify patients with depressive disorders/symptoms. Egger's test served for detecting the presence of publication bias. ${ }^{27} \mathrm{~A}$ value of $\mathrm{p}<0.10$ on Egger's test was considered indicative of statistically significant publication bias. Heterogeneity was evaluated by the $\chi^{2}$ test on Cochran's $Q$ statistic, ${ }^{28}$ which was quantified by $I^{2}$ values. The $I^{2}$ statistic estimates the percentage of total variation across studies due to true between-study differences rather than chance. In general, $I^{2}$ values greater than $60 \%-70 \%$ indicate the presence of substantial heterogeneity. ${ }^{29}$ Inter-rater agreements between investigators for study inclusion and methodological quality assessment were assessed using Cohen's $\kappa$ coefficient. ${ }^{30}$

\section{Patient and public involvement}

Patients or the public were not involved in the design, conduct, reporting or dissemination of our research.

\section{RESULTS}

\section{The review process and study characteristics}

We initially identified 890 records and finally retained 11 full texts (13 prevalence data) in the meta-analysis (online supplemental figure 1). ${ }^{31-41}$ Agreement between investigators on selection based on title and abstract was $\kappa=0.88$ and $\kappa=1.0$ for final inclusion.

Of the 11 included studies, 8 studies used nonprobabilistic sampling while 3 used probabilistic sampling. All studies prospectively collected and analysed data and used the same method to identify patients with depressive disorders. Sample size was adequate in nine studies and response rate in two studies (online supplemental table 2). Three studies had low risk of bias and eight moderate risk. None of the studies had high risk of bias.

The characteristics of included studies are presented in the table 1. All studies were cross-sectional. Patient Health Questionnaire-9 was the most used tool, $n=4$. The mean age varied between 50.3 and 59.6 years. The proportion of men varied between $28 \%$ and $54 \%$. Data on depressive disorders/symptoms were collected between 2002 and 2017. Four studies were conducted in South Africa, three studies in Nigeria, one study in Ghana, one study in both Ghana and Nigeria, one study in Ethiopia, and one study in Burkina Faso. None of the studies was conducted in Central Africa and North Africa. Talking about the language of the tool used to assess depressive status, four studies indicated that they used native/local languages back translated in English for reporting. ${ }^{32} 333536$

\section{Prevalence of depressive disorders/symptoms in people with} hypertension in Africa

In total, 5299 participants with hypertension were included. There was substantial heterogeneity for all analyses, all $I^{2} 35 \%$ (figure 1). The prevalence of depressive disorders was $18.6 \%$ (95\% CI $13.8 \%$ to $23.9 \%$; five studies) (figure 1). The adjusted prevalence taking into account the variance between diagnostic tools was $17.9 \%$ (95\% CI $13.0 \%$ to $23.4 \%$ ) with $52.7 \%$ of variance due to difference between tools. There was no publication bias $(p=0.789)$. There were no data on major depressive disorders.

The prevalence of depressive symptoms was $37.3 \%$ ( $95 \%$ CI $19.3 \%$ to $57.3 \%$; six studies). The adjusted prevalence taking into account the variance between diagnostic tools was $33.3 \%$ (95\% CI $9.9 \%$ to $61.6 \%$ ) with $74.1 \%$ of variance due to difference between tools. There was no publication bias $(\mathrm{p}=0.115)$.

The prevalence of major depressive symptoms was $7.9 \%$ (95\% CI $1.7 \%$ to $17.9 \%$; two studies). The adjusted prevalence taking into account the variance between diagnostic tools was $7.8 \%$ (95\% CI 3.0\% to $14.5 \%$ ) with $43.3 \%$ of variance due to difference between tools. The $p$ value on Egger's test was 0.789.

In subgroup analysis, there was no difference between population-based and hospital-based studies for all outcomes except for major depressive disorders where the prevalence was higher in population-based studies (12.6\%; $95 \%$ CI $8.8 \%$ to $17.0 \%$; one study) compared with hospital-based studies $(4.2 \%$; $95 \%$ CI $2.5 \%$ to $6.5 \%$; one study), $\mathrm{p}=0.0001$ (online supplemental figures 2, 3 and 4). However, there was a low number of studies in the groups compared. The prevalence of depressive disorders was higher among women $(23.8 \%$; $95 \%$ CI $18.7 \%$ to $29.3 \%$; one study) compared with men $(14.5 \%$; $95 \%$ CI $9.3 \%$ to $20.6 \%$; one study), $\mathrm{p}=0.0227$ (online supplemental figure 5). There was no difference in depressive symptoms (online supplemental figure 6).

\section{DISCUSSION}

This meta-analysis of data from 5299 adults with hypertension living in five countries in Africa revealed that depressive disorders and symptoms are prevalent in this population with substantial heterogeneity according to the diagnostic tools. This systematic review suggests that approximately one of five and a third of patients with hypertension have, respectively, depression and depressive symptoms.

Globally, there are dissimilarities between our findings and previous studies on depression among adults with hypertension. For instance, in China, a meta-analysis of 41 studies on the prevalence of depression in patients with hypertension found a higher rate of depression with a pooled prevalence of $26.9 \%$ (95\% CI $21.7 \%$ to $32.3 \%){ }^{10}$ Our review revealed a higher prevalence of depression 

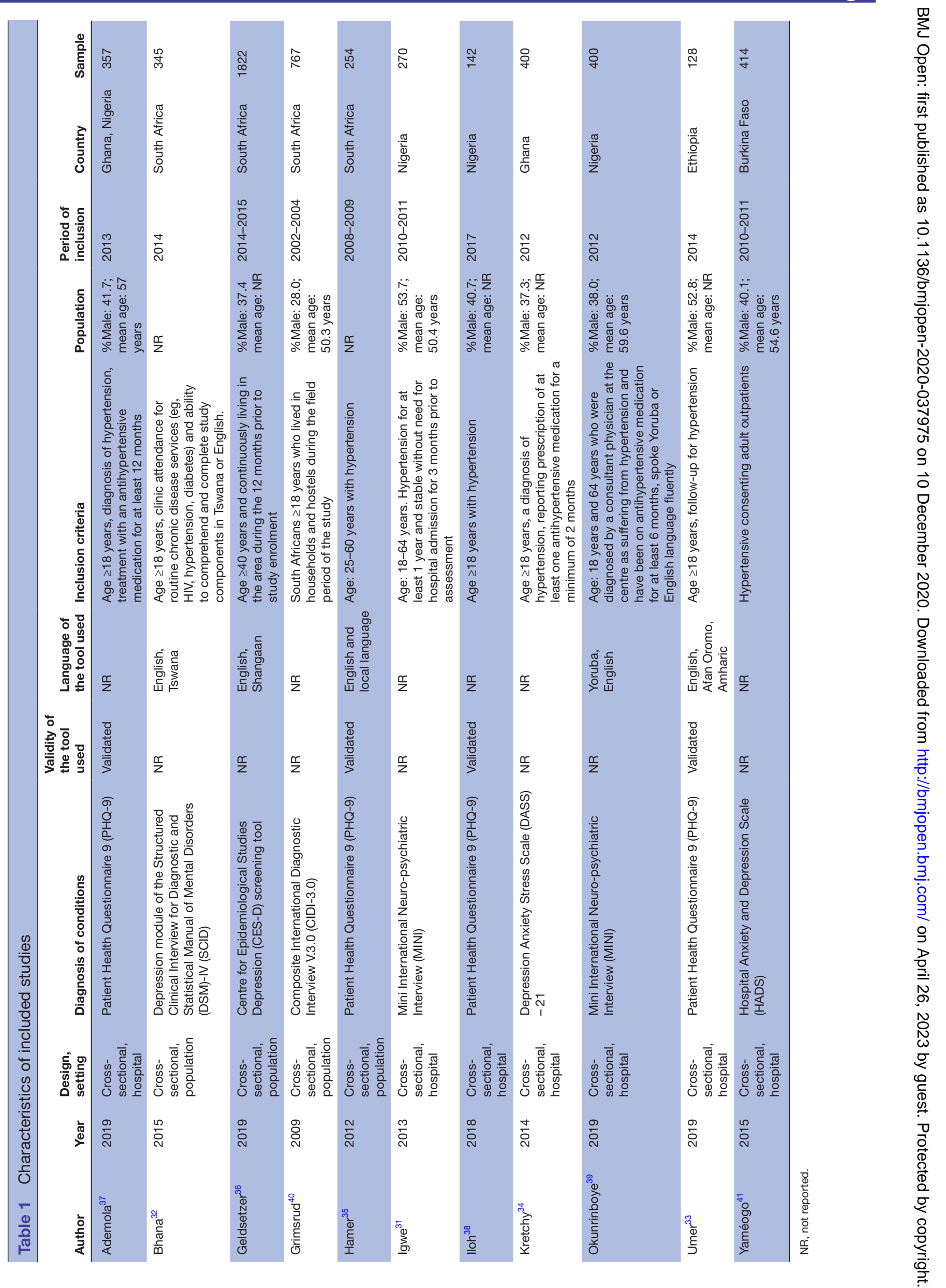


\section{A. Depressive disorders}

\begin{tabular}{lrc} 
Bhana, 2015 & 34 & 345 \\
Yaméogo, 2015 & 69 & 414 \\
Okunrinboye, 2019 & 81 & 400 \\
Grimsrud, 2009 & 163 & 767 \\
lgwe, 2013 & 72 & 270 \\
Subgroup prevalence & \multicolumn{2}{c}{$\mathbf{2 1 9 6}$} \\
Heterogeneity: $l^{2}=89.2 \%, p<0.0001$
\end{tabular}

\begin{tabular}{lrr} 
B. Depressive symptoms & \\
Kretchy, 2014 & 39 & 400 \\
Geldsetzer, 2019 & 317 & 1822 \\
Ademola, 2019 & 62 & 237 \\
Ademola, 2019 & 50 & 120 \\
Umer, 2019 & 69 & 128 \\
Iloh, 2018 & 118 & 142 \\
Subgroup prevalence & \multicolumn{2}{c}{$\mathbf{2 8 4 9}$} \\
Heterogeneity: $I^{2}=98.7 \%, p<0.0001$
\end{tabular}

\section{Major depressive symptoms}

\begin{tabular}{lrr} 
Kretchy, 2014 & 17 & 400 \\
Hamer, 2012 & 32 & 254 \\
Subgroup prevalence & \multicolumn{2}{c}{654} \\
Heterogeneity: $l^{2}=93.3 \%, p=0.0001$
\end{tabular}
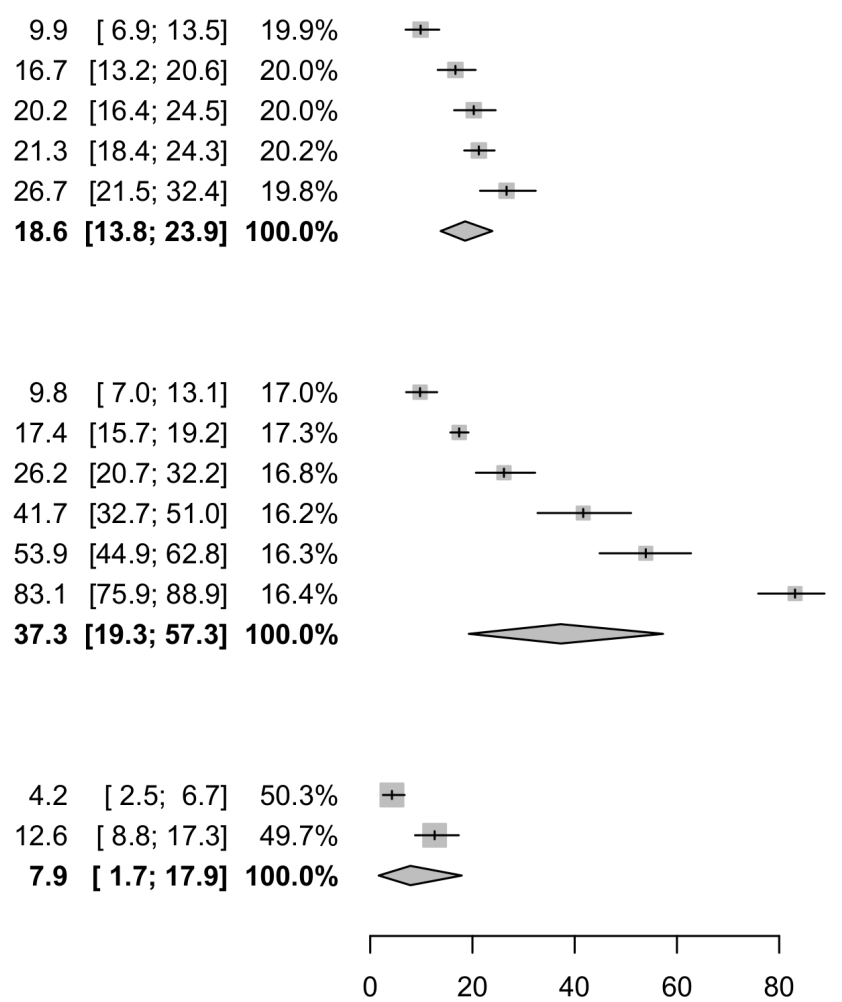

Figure 1 Crude prevalence of depressive disorders/symptoms in people living with hypertension in Africa.

among patients with hypertension compared with occidental studies. For instance, considering data from the US Multi-Disciplinary Group Practice Observational Study which included 4362 adult patients with hypertension, $13 \%$ had anxiety and/or depression. ${ }^{42}$ A cross-sectional study in Spain among 5954 patients with hypertension with high cardiovascular risk factors found that $15.6 \%$ of them had depression. ${ }^{19}$ These differences across regions can be explained by the changeability pertaining to the criteria and/or diagnostic tools used to screen depressive disorder. ${ }^{43}{ }^{44}$ Noteworthily, Li and colleagues who studied depressive disorder among patients with hypertension in a meta-analysis of 41 studies suggested that self-assessed screening tools of depression or depressive symptoms might overestimate the prevalence of depression. ${ }^{10}$ Indeed, they found $30 \%$ prevalence of depression using self-administrated diagnostic scales versus $21 \%$ prevalence using clinical interview tools. ${ }^{10}$ This could be linked to patients' confusion about symptoms possibly encountered in both depression and hypertension such as poor appetite, fatigue and sleep disturbances. ${ }^{10}$ In our review, the most used diagnostic tool was the Patient Health Questionnaire 9 (PHQ-9). Another fact to highlight is that differences in prevalence can also be explained by the cut-off points used for the same tool to define positive screening for depression. ${ }^{32} 4546$ Mahmood and colleagues, while assessing depression among 411 outpatients with hypertension in a hospital in Pakistan, using PHQ-9 with a score of 10 or above as the cut-off point, found a prevalence of $40.1 \%{ }^{47}$ which is twofold higher compared with our findings. In our study, we had substantial heterogeneity for all analyses which can also be related to the variance between diagnostic tools used for assessment of depression. The previously cited metaanalysis by $\mathrm{Li}$ and colleagues also showed evidence of high-level heterogeneity due to diagnostic tools considered in original studies. ${ }^{10}$

In our subgroup analysis, we found that there was no difference between population-based and hospitalbased studies for all outcomes except for major depressive disorders, for which the prevalence was higher in population-based studies. This result has to be cautiously interpreted since only two studies that used different tools in two distinct populations were compared. We also found that the prevalence of depressive disorders was higher among women compared with men. This finding is in accordance with what is known about gender differences in depression. ${ }^{48-50}$ This can be linked to hormonal differences between the two genders, and the fact that women experience periods of physiological changes such as menstruation, pregnancy and perimenopause. ${ }^{49} 5152$ There is growing evidence of the potential positive role of hormone replacement therapy on postmenopausal depression. ${ }^{51} 52$ The higher prevalence of depression and depressive symptoms in women compared to men in sub-Saharan African context can also be explained by cultural habits where men lesser express their psychological distress. ${ }^{53-55}$ 
This meta-analysis highlights the fact that depressive disorder is more frequently encountered among patients with hypertension compared with the general population. This review might substantiate the need to conduct further studies with an aim to investigate the tool for a better diagnosis of depression among patients with hypertension in order to reduce heterogeneity of results. Moreover, our review could justify carrying out epidemiological studies on relationship between depression and hypertension in other African regions in order to have a more representative regional picture of the evidence. Since we have been able to identify only 11 studies in the last 20 years, our study calls for more primary research on the relationship between hypertension and mental health in the continent, by using homogeneous diagnostic tools. Researchers, clinicians and public health policy makers can also explore implementation of registries to better measure the burden of mental health disorders in the continent. ${ }^{56-58}$ All this might help to establish the adapted policies pertaining to the management of patients with hypertension with depression, notably for a tailored pharmacological treatment. ${ }^{1859} 60$ Nevertheless, our study highlights that clinicians should be aware of the necessity to screen patients with hypertension for symptoms of depression, especially since previously published works have found that comorbid depression contributes to more deleterious cardiovascular outcomes. ${ }^{810151618} \mathrm{~A}$ meta-analysis of prospective cohort studies has suggested that people with depressive disorders had a higher risk of hypertension. ${ }^{15}$ Therefore, implementing strategies to reduce the burden of depressive disorders could help to reduce the prevalence of hypertension. Although pharmacological interventions can help to reduce the burden of depressive disorders, ${ }^{61}$ cost-effective nonpharmacological interventions should be explored first in the context of resource-limited settings like most of the countries in Africa. ${ }^{62} 63$

This study should however be interpreted considering some limitations. First we found a huge heterogeneity between studies for which we undertook a subgroup analysis to investigate sources of heterogeneity and adjusted analysis to take into account the variance due to diagnostic tools. However, some characteristics that may further explain heterogeneity were not reported or there were not enough studies to conduct such analysis including subregions and age groups. Second, there was substantial variability regarding the representativeness of regions and countries, with some less represented or not represented. This may weaken the generalisability of our findings and call for more epidemiological studies in this region. Not all studies had low risk of bias, especially, most of the studies used non-probabilistic sampling. However, due to the low number of studies included in this metaanalysis, we were not able to perform sensitivity analysis to assess the robustness of our findings based on methodological quality.

Despite these limitations, this first systematic review and meta-analysis on depressive disorders/symptoms in people living with hypertension in Africa provided a clear summary of the existing knowledge. This systematic review is the starting point for understanding the epidemiology of and relationship between mental health and hypertension in African countries where it is challenging to collect such data. A protocol had been registered before, and we used rigorous methodological and statistical procedures to obtain and pool data. Furthermore, we have taken into account the variability due to diagnostic tools. There was no publication bias.

\section{CONCLUSIONS}

Overall, our review found that depressive disorders and symptoms are prevalent in people living with hypertension in selected African countries. Including an assessment of mental health in patients with hypertension seems prudent, with the potential for intervention. However, since our analysis has limitations pertaining to consistency of diagnostic tools within studies and also to the unrepresentative geographical distribution, further studies would be relevant in order to reinforce our findings. All this could be a support for personalised management of patients with hypertension and depression.

\section{Twitter Jean Joel Bigna @BIGNARIM}

Contributors Conception, designing of the protocol, studies selection, data extraction, writing of the first draft: JJB, FTE. Literature search, data management, data synthesis and analysis: JJB. Critical revision and approval of the final version: FTE, TNM, JJB. Guarantor of the review: JJB.

Funding The authors have not declared a specific grant for this research from any funding agency in the public, commercial or not-for-profit sectors.

Competing interests None declared.

Patient consent for publication Not required.

Provenance and peer review Not commissioned; externally peer reviewed.

Data availability statement All data relevant to the study are included in the article or uploaded as supplemental information.

Supplemental material This content has been supplied by the author(s). It has not been vetted by BMJ Publishing Group Limited (BMJ) and may not have been peer-reviewed. Any opinions or recommendations discussed are solely those of the author(s) and are not endorsed by BMJ. BMJ disclaims all liability and responsibility arising from any reliance placed on the content. Where the content includes any translated material, BMJ does not warrant the accuracy and reliability of the translations (including but not limited to local regulations, clinical guidelines, terminology, drug names and drug dosages), and is not responsible for any error and/or omissions arising from translation and adaptation or otherwise.

Open access This is an open access article distributed in accordance with the Creative Commons Attribution Non Commercial (CC BY-NC 4.0) license, which permits others to distribute, remix, adapt, build upon this work non-commercially, and license their derivative works on different terms, provided the original work is properly cited, appropriate credit is given, any changes made indicated, and the use is non-commercial. See: http://creativecommons.org/licenses/by-nc/4.0/.

\section{ORCID iDs}

Temgoua Ngou Mazou http://orcid.org/0000-0002-2476-3550

Jean Joel Bigna http://orcid.org/0000-0001-8018-6279

\section{REFERENCES}

1 GBD 2015 Risk Factors Collaborators. Global, regional, and national comparative risk assessment of 79 behavioural, environmental and occupational, and metabolic risks or clusters of risks, 1990-2015: 
a systematic analysis for the global burden of disease study 2015 . Lancet 2016;388:1659-724.

2 Kearney PM, Whelton M, Reynolds K, et al. Global burden of hypertension: analysis of worldwide data. Lancet 2005;365:217-23.

3 Lawes CMM, Vander Hoorn S, Rodgers A, et al. Global burden of blood-pressure-related disease, 2001. Lancet 2008;371:1513-8.

4 World Health Organization. Hypertension, 2019. Available: https:// www.who.int/news-room/fact-sheets/detail/hypertension [Accessed 16 Feb 2019].

5 ZhouB, DanaeiG, StevensGA, et al. Long-Term and recent trends in hypertension awareness, treatment, and control in 12 high-income countries: an analysis of 123 nationally representative surveys. Lancet 2019;394:639-51.

6 Arima H, Barzi F, Chalmers J. Mortality patterns in hypertension. $J$ Hypertens 2011;29 Suppl 1:S3-7.

7 Zhang D, Wang G, Zhang P, et al. Medical expenditures associated with hypertension in the U.S., 2000-2013. Am J Prev Med 2017;53:S164-71.

8 Scalco AZ, Scalco MZ, Azul JBS, et al. Hypertension and depression. Clinics 2005;60:241-50.

9 Ngo VK, Rubinstein A, Ganju V, et al. Grand challenges: integrating mental health care into the non-communicable disease agenda. PLoS Med 2013;10:e1001443.

$10 \mathrm{Li} \mathrm{Z,} \mathrm{Li} \mathrm{Y,} \mathrm{Chen} \mathrm{L,} \mathrm{et} \mathrm{al.} \mathrm{Prevalence} \mathrm{of} \mathrm{depression} \mathrm{in} \mathrm{patients} \mathrm{with}$ hypertension. Medicine 2015;94:e1317.

11 Rehm J, Shield KD. Global burden of disease and the impact of mental and addictive disorders. Curr Psychiatry Rep 2019;21:10.

12 Ferrari AJ, Charlson FJ, Norman RE, et al. Burden of depressive disorders by country, sex, age, and year: findings from the global burden of disease study 2010. PLoS Med 2013;10:e1001547.

13 World Health Organization. Depression and other common mental disorders. Geneva: World Health Organization, 2017.

14 Schaakxs R, Comijs HC, van der Mast RC, et al. Risk factors for depression: differential across age? Am J Geriatr Psychiatry 2017;25:966-77.

15 Meng L, Chen D, Yang Y, et al. Depression increases the risk of hypertension incidence: a meta-analysis of prospective cohort studies. J Hypertens 2012;30:842-51.

16 Song X, Zhang Z, Zhang R, et al. Predictive markers of depression in hypertension. Medicine 2018;97:e11768.

17 Sandström YK, Ljunggren G, Wändell P, et al. Psychiatric comorbidities in patients with hypertension--a study of registered diagnoses 2009-2013 in the total population in Stockholm County, Sweden. J Hypertens 2016;34:414-20.

18 Graham N, Ward J, Mackay D, et al. Impact of major depression on cardiovascular outcomes for individuals with hypertension: prospective survival analysis in UK Biobank. BMJ Open 2019;9:e024433.

19 Mejia-Lancheros C, Estruch R, Martínez-González MA, et al. Blood pressure values and depression in hypertensive individuals at high cardiovascular risk. BMC Cardiovasc Disord 2014;14:109.

20 Xue J, Chen S, Bogner HR, et al. The prevalence of depressive symptoms among older patients with hypertension in rural China. Int $J$ Geriatr Psychiatry 2017;32:1411-7.

21 Munn Z, Moola S, Lisy K, et al. Chapter 5: Systematic reviews of prevalence and incidence. In: Aromataris E, Munn Z, eds. JBI manual for evidence synthesis. Adelaide: The Joanna Briggs Institute, 2020: $175-215$

22 Liberati A, Altman DG, Tetzlaff J, et al. The PRISMA statement for reporting systematic reviews and meta-analyses of studies that evaluate healthcare interventions: explanation and elaboration. BMJ 2009;339:b2700.

23 American Psychiatric Association, DSM-IV APATF. Diagnostic and statistical manual of mental disorders, fourth edition: DSM-IV-TR®; 2000: 996.

24 American Psychiatric Pub. Diagnostic and statistical manual of mental disorders (DSM-5®); 2013: 1414

25 World Health Organization. The ICD-10 classification of mental and behavioural disorders: clinical descriptions and diagnostic guidelines; 1992: 377p

26 Barendregt JJ, Doi SA, Lee YY, et al. Meta-Analysis of prevalence. J Epidemiol Community Health 2013;67:974-8.

27 Egger M, Davey Smith G, Schneider M, et al. Bias in meta-analysis detected by a simple, graphical test. BMJ 1997;315:629-34.

28 Cochran WG. The combination of estimates from different experiments. Biometrics 1954;10:101-29.

29 Higgins JPT, Thompson SG. Quantifying heterogeneity in a metaanalysis. Stat Med 2002;21:1539-58.

30 Viera AJ, Garrett JM. Understanding interobserver agreement: the kappa statistic. Fam Med 2005;37:360-3.
31 Igwe MN, Uwakwe R, Ahanotu CA, et al. Factors associated with depression and suicide among patients with diabetes mellitus and essential hypertension in a Nigerian teaching hospital. Afr Health Sci 2013;13:68-77.

32 Bhana A, Rathod SD, Selohilwe O, et al. The validity of the patient health questionnaire for screening depression in chronic care patients in primary health care in South Africa. BMC Psychiatry 2015;15:118.

33 Umer H, Negash A, Birkie M, et al. Determinates of depressive disorder among adult patients with cardiovascular disease at outpatient cardiac clinic Jimma university teaching Hospital, South West Ethiopia: cross-sectional study. Int J Ment Health Syst 2019;13:13

34 Kretchy IA, Owusu-Daaku FT, Danquah SA. Mental health in hypertension: assessing symptoms of anxiety, depression and stress on anti-hypertensive medication adherence. Int J Ment Health Syst 2014;8:25.

35 Hamer M, Frasure-Smith N, Lespérance F, et al. Depressive symptoms and 24-hour ambulatory blood pressure in Africans: the SABPA study. Int J Hypertens 2012;2012:1-6.

36 Geldsetzer P, Vaikath M, Wagner R, et al. Depressive symptoms and their relation to age and chronic diseases among middle-aged and older adults in rural South Africa. J Gerontol A Biol Sci Med Sci 2019;74:957-63.

37 Ademola AD, Boima V, Odusola AO, et al. Prevalence and determinants of depression among patients with hypertension: a cross-sectional comparison study in Ghana and Nigeria. Niger J Clin Pract 2019;22:558-65.

38 Iloh GUP, Aguocha GU, Amadi AN, et al. Depression among ambulatory adult patients in a primary care clinic in southeastern Nigeria. Niger Postgrad Med J 2018;25:204-12.

39 Okunrinboye HI, Otakpor AN, Ilesanmi OS. Depression and medication-adherence in patients with hypertension attending a tertiary health facility in south-west Nigeria. Pan Afr Med J 2019;33:27.

40 Grimsrud A, Stein DJ, Seedat S, et al. The association between hypertension and depression and anxiety disorders: results from a nationally-representative sample of South African adults. PLoS One 2009;4:e5552.

41 Yaméogo NV, Samadoulougou A, Kagambèga LJ, et al. Sleep disorders, anxiety and depressive symptoms and cardiovascular risk among hypertensive black Africans: cross-sectional study of 414 hypertensive followed as outpatients at the central university hospital of Ouagadougou (Burkina Faso). Pan African Medical Journal 2015;21:185-96

42 Ho AK, Thorpe CT, Pandhi N, et al. Association of anxiety and depression with hypertension control: a US multidisciplinary group practice observational study. J Hypertens 2015;33:2215-22.

43 Levis B, Yan XW, He C, et al. Comparison of depression prevalence estimates in meta-analyses based on screening tools and rating scales versus diagnostic interviews: a meta-research review. BMC Med 2019;17:65.

44 de Joode JW, van Dijk SEM, Walburg FS, et al. Diagnostic accuracy of depression questionnaires in adult patients with diabetes: a systematic review and meta-analysis. PLoS One 2019;14:e0218512.

45 Manea L, Gilbody S, McMillan D. Optimal cut-off score for diagnosing depression with the patient health questionnaire (PHQ-9): a meta-analysis. CMAJ 2012;184:E191-6.

46 Levis B, Benedetti A, Thombs BD, et al. Accuracy of patient health Questionnaire-9 (PHQ-9) for screening to detect major depression: individual participant data meta-analysis. BMJ 2019;365:I1476.

47 Mahmood S, Hassan SZ, Tabraze M, et al. Prevalence and predictors of depression amongst hypertensive individuals in Karachi, Pakistan. Cureus 2017;9:e1397.

48 Abate KH. Gender disparity in prevalence of depression among patient population: a systematic review. Ethiop J Health Sci 2013;23:283-8

49 Salk RH, Hyde JS, Abramson LY. Gender differences in depression in representative national samples: meta-analyses of diagnoses and symptoms. Psychol Bull 2017;143:783-822.

50 Picco L, Subramaniam M, Abdin E, et al. Gender differences in major depressive disorder: findings from the Singapore mental health study. Singapore Med J 2017;58:649-55.

51 Albert PR. Why is depression more prevalent in women? J Psychiatry Neurosci 2015;40:219-21.

52 Bromberger JT, Epperson CN. Depression during and after the Perimenopause: impact of hormones, genetics, and environmental determinants of disease. Obstet Gynecol Clin North Am 2018;45:663-78.

53 Call JB, Shafer K. Gendered manifestations of depression and help seeking among men. Am J Mens Health 2018;12:41-51. 
54 Bailey RK, Mokonogho J, Kumar A. Racial and ethnic differences in depression: current perspectives. Neuropsychiatr Dis Treat 2019;15:603-9.

55 Ikram UZ, Snijder MB, de Wit MAS, et al. Perceived ethnic discrimination and depressive symptoms: the buffering effects of ethnic identity, religion and ethnic social network. Soc Psychiatry Psychiatr Epidemiol 2016;51:679-88.

56 Prakash J, Ramakrishnan T, Das RC, et al. Central registry in psychiatry: a structured review. Ind Psychiatry J 2014;23:10-14.

57 Yang M, Loeb DF, Sprowell AJ, et al. Design and implementation of a depression Registry for primary care. Am J Med Qual 2019;34:59-66.

58 Stewart R, Davis K. 'Big data' in mental health research: current status and emerging possibilities. Soc Psychiatry Psychiatr Epidemiol 2016;51:1055-72.

59 Peixoto MF, Cesaretti M, Hood SD, et al. Effects of SSRI medication on heart rate and blood pressure in individuals with hypertension and depression. Clin Exp Hypertens 2019;41:428-33.
60 Offidani E, Benasi G, Charlson ME, et al. Impact of depression and Demoralization on blood pressure control in African Americans with hypertension: findings from the triumph trial. J Racial Ethn Health Disparities 2018;5:913-8.

61 Cipriani A, Furukawa TA, Salanti G, et al. Comparative efficacy and acceptability of 21 antidepressant drugs for the acute treatment of adults with major depressive disorder: a systematic review and network meta-analysis. Lancet 2018;391:1357-66.

62 Sukhato K, Lotrakul M, Dellow A, et al. Efficacy of home-based nonpharmacological interventions for treating depression: a systematic review and network meta-analysis of randomised controlled trials. BMJ Open 2017;7:e014499.

63 Frost R, Bauernfreund Y, Walters K. Non-Pharmacological interventions for depression/anxiety in older adults with physical comorbidities affecting functioning: systematic review and metaanalysis. Int Psychogeriatr 2019;31:1121-36. 